New results

\title{
Mathematical Model of Mechanical Virion-Cell Interaction During Early Engulfment in HIV
}

Elizabeth Kruse $^{1}$, Tamer Abdalrahman ${ }^{1}$, Philippe Selhorst ${ }^{2,3,4}$, Thomas Franz ${ }^{1,5^{*}}$

${ }^{1}$ Division of Biomedical Engineering, Department of Human Biology, University of Cape Town, South Africa

${ }^{2}$ Division of Virology, Department of Pathology, University of Cape Town, South Africa

${ }^{3}$ Institute of Infectious Diseases and Molecular Medicine, University of Cape Town, South Africa

${ }^{4}$ Virology Unit, Department of Biomedical Sciences, Institute of Tropical Medicine, Antwerp, Belgium

${ }^{5}$ Bioengineering Science Research Group, Engineering Sciences, University of Southampton, UK

*Corresponding author Thomas Franz

Department of Human Biology

Faculty of Health Sciences

University of Cape Town

Private Bag X3

Observatory 7935

Email: Thomas.franz@uct.ac.za 


\begin{abstract}
While HIV entry into host cells has been extensively studied from a biological and biochemical perspective, the influence of mechanical parameters of virions and cells on engulfment and invagination is not well understood. The present work aimed at developing a mathematical model to quantify effects of mechanical and morphological parameters on engulfment forces and energies of HIV particles. Invagination force and engulfment energy were described as analytical functions of radius and elastic modulus of virion and cell, ligand-receptor energy density, receptor complex density, and engulfment depth for early stage engulfment. The models were employed to study the effects of (a) virion-membrane contact geometry on required invagination force for global cell geometries and ultrastructural cell membrane features, and (b) virion radius and number of gp120 proteins on engulfment energy. The invagination force was equal for cells of various sizes (i.e. macrophages and lymphocytes), but lower when considering ultrastructural membrane. The magnitude of the normalised engulfment energy was higher for a mature than for an immature, larger virion with the same number of 72 gp120 spikes, but it decreased for a mature virion with a reduced number of gp120 spikes. The results suggest that for early stage engulfment (1) localised cell membrane features promote invagination and may play a role in entry ability, and (2) shedding of gp120 proteins during maturation reduces engulfment energy which is expected to reduce entry ability.
\end{abstract}

Keywords: endocytosis; engulfment energy; entry ability; human immunodeficiency virus; virion mechanics; elastic modulus; stiffness 


\section{Introduction}

The mechanism of HIV entry into host cells has been extensively studied, however there is limited research on how mechanical factors influence this entry process. Mathematical modelling of the mechanical interactions between virion and cell membrane allows for a quantification of forces and energies involved in virion engulfment and can as such provide data that are not easily accessible with experimental approaches.

Mathematical models have been used to investigate how contact force, mechanical work, and pressure varied with engulfment depth of the virion (Gefen, 2010; Sun and Wirtz, 2006). Gefen (2010) studied the impact of virion size and cell stiffness on the forces, work and pressures during virion engulfment, limited to small cellular deformations. Both studies assumed a uniform global radius of the host cell (which is very large compared to the radius of the virion) and did not account for morphological irregularities on the cellular surface at micro- or nanoscale. However, electron microscopy images of the HIV-cellular interaction (Gentile et al., 1994) reveal a cellular surface with local curvatures in the nanometre range. Such localised surface features of the cell membrane may indeed play a role in the virion-cell interactions during engulfment and endocytosis and the likelihood of viral infection of a cell.

It has been reported that mature HIV particles have a higher entry ability into host cells compared to immature virions (Murakami et al., 2004; Wyma et al., 2004). Kol et al. (2007) demonstrated that virion stiffness is reduced during the maturation process. Pang et al. (2013) demonstrated that the reduced stiffness of the virions resulted in an increased entry ability of the virions into the cells. Based on the physical interaction between virion and cell membrane, this suggests that contact mechanics plays a large role during early virion-cell interactions.

The aim of the current study is to develop a mathematical model that allows to investigate the sensitivity of the mechanics of early virion engulfment to changes of morphological and mechanical parameters of HIV virions and host cells to guide future experimental studies.

\section{Methods}

The virion engulfment model describes the engulfment energy and the invagination force and was implemented in MATLAB R2014a (Mathworks Inc, Natick, MA, USA). The model is 
based on continuum models for receptor-mediated endocytosis of viruses that employ contact mechanics and consider ligand-receptor complex formation energy (Gefen, 2010; Sun and Wirtz, 2006).

Our model assumes a virion-cell arrangement as illustrated schematically in Figure 1. The process of infection is initiated when the virion ligands (i.e. gp120) dock to the cell receptors (i.e. CD4), the cell starts to engulf a virion (with a radius very small compared to that of the cell, i.e. $R_{v}<<R_{c}$ ) to an engulfment depth $d$ by generating an invagination force $F$. Due to the large size difference between cell and virion, the cell membrane is approximated as a flat surface. This engulfment process is a critical initial step in triggering fusion between the virion and cell membrane and establishing infection of the host cell.

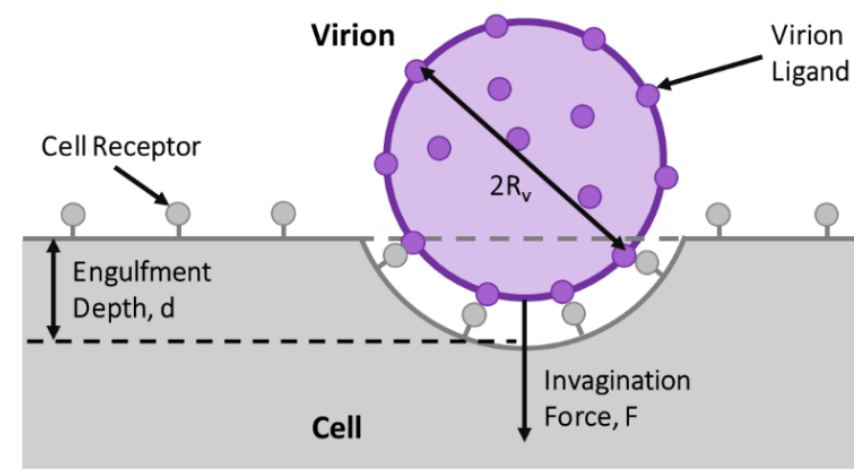

Figure 1: Schematic of virion-cell membrane interaction during virion engulfment.

\subsection{Engulfment Energy}

The total energy $\left(W_{T}\right)$ of the engulfment process comprises three energy terms; namely the adhesion energy $\left(W_{1}\right)$, the membrane energy before and after bending during the engulfment process $\left(W_{2}\right)$, and the elastic energy of the cytoskeleton deformation $\left(W_{3}\right)$ :

$$
W_{T}=W_{1}+W_{2}+W_{3} .
$$

The adhesion energy gained from ligand-receptor binding, Eq. (2), is utilised to deform the cell membrane, thereby driving the entry process:

$$
W_{1}=-\varphi A .
$$

Here, $\varphi$ is the ligand-receptor energy density function and $A$ is the area of contact between the virion and the cell. This area can be calculated as the contact area between a sphere and a half-space and is a function of the engulfment depth, $d$, and the virion radius, $R_{v}$, Eq. (3) 
(Johnson, 1985). The radius of a mature and immature HIV virion was taken as $55 \mathrm{~nm}$ and 73 $\mathrm{nm}$, respectively (Gentile et al., 1994):

$$
A=2 \pi R_{v} d .
$$

The receptor-ligand density function $\varphi$ depends on the energy gained per receptor-ligand complex $(f)$ and the receptor complex density $(\rho)$ :

$$
\varphi=f \rho .
$$

The energy gained $(f)$ is $10 k_{B} T$ (Sun and Wirtz, 2006), where $k_{B}$ is the Boltzmann constant $\left(1.3807 \times 10^{-23} \mathrm{~J} / \mathrm{K}\right)$ and $T$ is the absolute temperature which is taken to be $310 \mathrm{~K}$ for the human body (Gefen, 2010). The receptor complex density $(\rho)$ is determined by the ratio of the total number of glycoproteins and the virion surface area $\left(4 \pi R_{v}^{2}\right)$. For HIV, each gp120 spike consists of 3 glycoproteins. Therefore, an immature HIV virion with 72 spikes (Gelderblom, 1991; Grief et al., 1989) has 216 glycoproteins, and a mature HIV virion with 10 spikes (Layne et al., 1992) has 30 glycoproteins.

The second energy term, $W_{2}$, describes the membrane energy before and after bending. This assumes that purely elastic energy is present and does not account for the viscoelasticity of the cytoskeleton and the heterogeneity of the cell. This energy is well described by the Canham-Helfrich theory (Canham, 1970; Helfrich, 1973), and is defined as:

$$
W_{2}=\frac{\pi k d}{R_{v}}+\gamma \pi d^{2} .
$$

Here, $k$ is the bending modulus of the cell membrane which is taken to be $20\left(k_{B} T\right)$, and $\gamma$ is the cellular surface tension which is approximately $0.005\left(k_{B} T\right)$ (Sun and Wirtz, 2006).

The elastic energy of the cytoskeleton, $W_{3}$, is a function of the effective elastic modulus of the cell and the virus $\left(E^{*}\right)$, the effective radius $\left(R^{*}\right)$, and the engulfment depth $(d)$ :

$$
W_{3}=\frac{8}{15} E^{*} \sqrt{R^{*}} d^{\frac{5}{2}} .
$$

The normalised total engulfment energy is obtained as

$$
W_{\mathrm{Tn}}=\frac{W_{T}}{k_{B} T} .
$$

The effective elastic modulus (Eq. (8)) is a function of the elastic modulus of the cell $\left(E_{c}\right)$ which is $35 \mathrm{kPa}$ (Slomka et al., 2009), and the elastic modulus of HIV $\left(E_{v}\right)$ which is $440 \mathrm{MPa}$ for mature particles and $930 \mathrm{MPa}$ for immature particles. The Poisson's ratio of the HIV particle $\left(v_{v}\right)$ is 0.4 (Ahadi et al., 2013) and that of the cell $\left(v_{c}\right)$ is 0.5 (Sun and Wirtz, 2006). 


$$
E^{*}=\frac{E_{c} E_{v}}{\left(1-v_{v}^{2}\right) E_{c}+\left(1-v_{c}^{2}\right) E_{v}} .
$$

The effective radius $R^{*}$ is a function of the virion radius $\left(R_{v}\right)$ and the cell radius $\left(R_{c}\right)$ :

$$
R^{*}=\frac{R_{v} R_{C}}{R_{v}+R_{c}}
$$

For the cell radius, both the global radius of the cell (3-4 $\mu \mathrm{m}$ for lymphocytes and $7.5-40 \mu \mathrm{m}$ for macrophages) (Kierszenbaum and Tres, 2015) and curvature of the localised cell membrane features in the nanometre range (Gentile et al., 1994) were used.

\subsection{Invagination Force}

The force required for the invagination of the virus into the cell is defined using the Hertz model of frictionless contact between two spheres:

$$
F=\frac{4}{3} \sqrt{d^{3} R^{*}\left(E^{*}\right)^{2}}
$$

According to Dintwa et al. (2007) the assumption of frictionless contact is reasonable. A normalised engulfment depth $\alpha$ is used to relate the engulfment depth of the virus to the virus radius:

$$
\alpha=\frac{d}{2 R_{v}} \times 100 .
$$

As the Hertz model is accurate for small deformation only, predictions were limited to a normalised engulfment depth of $\alpha \leq 10 \%$ (Gefen, 2010).

\section{Results}

Figure 2 illustrates the required invagination force versus engulfment depth of mature HIV particles for various cell sizes. There is no discernible difference in the force required for invagination for the global cell radius of macrophages and lymphocytes (i.e. 4,000 and $40,000 \mathrm{~nm}$, respectively) which are both very large compared to the radius of the HIV particle. However, a considerable decrease of the invagination force is observed when local curvatures are considered that are the same order of magnitude as the virion radius. 


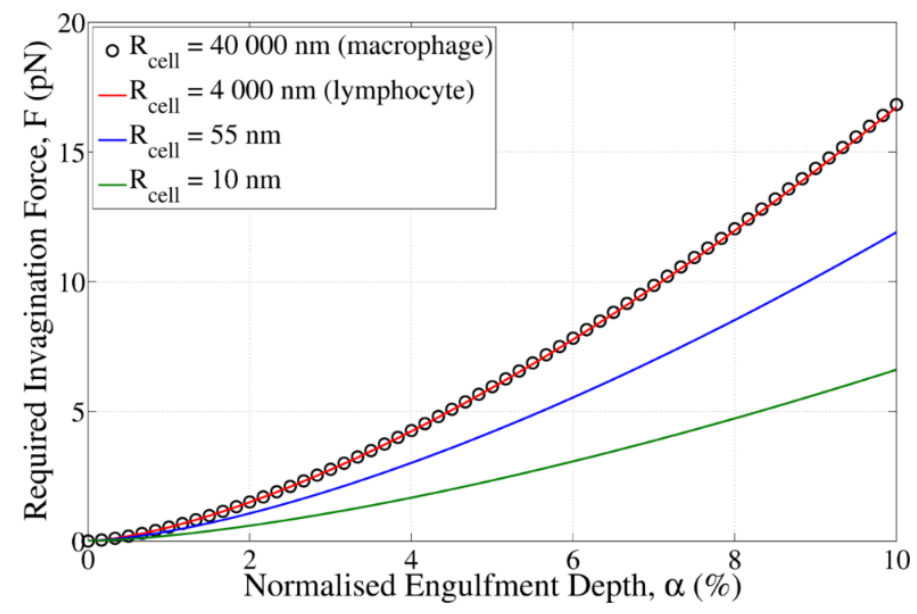

Figure 2: Required invagination force versus normalised engulfment depth for different radii of the cell membrane representing the global size of a macrophage $\left(R_{\text {cell }}=40,000 \mathrm{~nm}\right)$ and a lymphocyte $\left(R_{\text {cell }}=\right.$ $4,000 \mathrm{~nm}$ ) as well as localised membrane curvatures.

In Figure 3, the normalised total engulfment energy versus engulfment depth is illustrated for different HIV particles sizes and different numbers of gp120 spikes, representing particles of different maturity. For a decrease in the size of the virion from an immature virion with a radius of 73 to and a mature virion with a radius of $55 \mathrm{~nm}$, an increase in the absolute magnitude of the normalised total engulfment energy from $|-160.0|$ to $|-184.3|$ is predicted at an engulfment of $\alpha=10 \%$. However, when considering the reduced number of gp120 spikes as a result of maturation, the normalised energy value decreases from $|-184.3|$ (x) to |-90.95| (o) for an engulfment depth of $10 \%$, which is even lower than the engulfment energy for an immature virion.

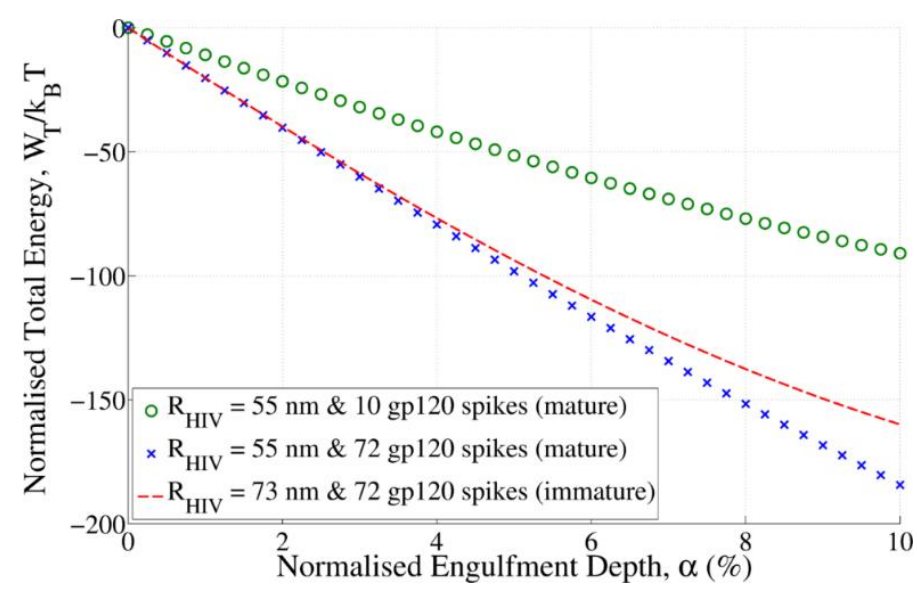

Figure 3: Normalised total engulfment energy versus normalised engulfment depth for HIV particles with different radius and number of gp120 spikes, respectively. 


\section{Discussion}

Analytical modelling was used to investigate the impact of certain parameters on the theoretical contact mechanics associated with the virion-cell interactions. The model determines the total engulfment energy and invagination force during the early engulfment phase.

The variation of the global cell radius, representing the difference in size between lymphocytes and macrophages, had very little impact on the required invagination force (Figure 2). This is in line with previous models that investigated the impact of cellular size and determined that the global cell radius has a minimal effect on the mechanics of the virion-cell interaction (Gefen, 2010).

However, the cell membrane exhibits local morphological irregularities at nanometre length scale (Gentile et al., 1994). These local surface morphologies with curvatures of the same order of magnitude as those of the virion affected the required invagination force considerably. Our results indicate that cell membrane morphologies with curvatures in the nanometre range substantially reduce the invagination force required during virion engulfment and are potential sites for easier virion entry. This suggests that the surface morphology of cells may play a role in the infection process and infectivity.

The maturation stage of virions had considerable effect on the engulfment energy. A previous study investigated the impact of virion size (Gefen, 2010), indicating an increase of the magnitude of the engulfment energy in agreement with our results (Figure 3). In addition, we found that the reduction in the number of gp120 spikes, associated with virion maturation, leads to a substantial reduction in magnitude of the engulfment energy, primarily due to a lower adhesion energy associated with gp120. The reduction in engulfment energy is likely to reduce the entry ability. This contradicts with previous observations that entry ability increases as virions mature (Jiang and Aiken, 2006; Kol et al., 2007). An explanation for this contradiction may be that other factors also determine entry ability e.g. conformational changes in gp120 during maturation that impact the functional capabilities of these ligands, and consequently the entry ability of the virion (Murakami et al., 2004; Wyma et al., 2004).

The present model has some limitations that should be taken into account. Firstly, the model does not consider viscoelastic phenomena of the virus or the cell. Secondly, as Eqs. 6-10 were derived based on the assumption of small-deformation elasticity, the model is only 
suitable for the representation of early invagination, hence, calculations were limited to $\alpha \leq$ $10 \%$.

Nevertheless, based on current model two mechanical physical parameters were identified as candidates for experimental investigations to determine their impact on virion engulfment, i.e. cell surface morphology and conformational changes in gp120 during maturation.

\section{Funding}

The work was supported by the National Research Foundation of South Africa (grant numbers UID92531 and UID93542), and the South African Medical Research Council (grant number SIR328148).

\section{Conflict of Interest}

The authors declare that they have no conflict of interest.

\section{References}

Ahadi, A., Johansson, D., Evilevitch, A., 2013. Modeling and simulation of the mechanical response from nanoindentation test of DNA-filled viral capsids. J Biol Phys 39, 183-199.

Canham, P.B., 1970. The minimum energy of bending as a possible explanation of the biconcave shape of the human red blood cell. J. Theor. Biol. 26, 61-81.

Dintwa, E., Tijskens, E., Ramon, H., 2007. On the accuracy of the Hertz model to describe the normal contact of soft elastic spheres. Granular Matter 10, 209-221.

Gefen, A., 2010. Effects of virus size and cell stiffness on forces, work, and pressures driving membrane invagination in a receptor-mediated endocytosis. J Biomech Eng 132, 084501.

Gelderblom, H.R., 1991. Assembly and morphology of HIV: potential effect of structure on viral function. AIDS 5, 617-637. 
Gentile, M., Adrian, T., Scheidler, A., Ewald, M., Dianzani, F., Pauli, G., Gelderblom, H.R., 1994. Determination of the Size of HIV Using Adenovirus Type-2 as an Internal Length Marker. J Virol Methods 48, 43-52.

Grief, C., Hockley, D.J., Fromholc, C.E., Kitchin, P.A., 1989. The morphology of simian immunodeficiency virus as shown by negative staining electron microscopy. J Gen Virol 70 ( Pt 8), 2215-2219.

Helfrich, W., 1973. Elastic Properties of Lipid Bilayers: Theory and Possible Experiments. Zeitschrift für Naturforschung C 28, 693-703.

Jiang, J., Aiken, C., 2006. Maturation of the viral core enhances the fusion of HIV-1 particles with primary human T cells and monocyte-derived macrophages. Virology 346, 460-468.

Johnson, K.L., 1985. Contact Mechanics.

Kierszenbaum, A.L., Tres, L., 2015. Histology and Cell Biology: An Introduction to Pathology, 4 ed. Saunders.

Kol, N., Shi, Y., Tsvitov, M., Barlam, D., Shneck, R.Z., Kay, M.S., Rousso, I., 2007. A stiffness switch in HIV. Biophys. J. 92, 1777-1783.

Layne, S.P., Merges, M.J., Dembo, M., Spouge, J.L., Conley, S.R., Moore, J.P., Raina, J.L., Renz, H., Gelderblom, H.R., Nara, P.L., 1992. Factors underlying spontaneous inactivation and susceptibility to neutralization of human immunodeficiency virus. Virology 189, 695714.

Murakami, T., Ablan, S., Freed, E.O., Tanaka, Y., 2004. Regulation of Human Immunodeficiency Virus Type 1 Env-Mediated Membrane Fusion by Viral Protease Activity. J. Virol. 78, 1026-1031.

Pang, H.B., Hevroni, L., Kol, N., Eckert, D.M., Tsvitov, M., Kay, M.S., Rousso, I., 2013. Virion stiffness regulates immature HIV-1 entry. Retrovirology 10, 4.

Slomka, N., Or-Tzadikario, S., Sassun, D., Gefen, A., 2009. Membrane-Stretch-Induced Cell Death in Deep Tissue Injury: Computer Model Studies. Cell Mol Bioeng 2, 118-132. 
Sun, S.X., Wirtz, D., 2006. Mechanics of enveloped virus entry into host cells. Biophys J 90, L10-12.

Wyma, D.J., Jiang, J., Shi, J., Zhou, J., Lineberger, J.E., Miller, M.D., Aiken, C., 2004.

Coupling of Human Immunodeficiency Virus Type 1 Fusion to Virion Maturation: a Novel Role of the gp41 Cytoplasmic Tail. J. Virol. 78, 3429-3435. 